\title{
DEVELOPMENT OF A PARTITIONED FINITE VOLUME-FINITE ELEMENT FLUID-STRUCTURE INTERACTION SCHEME FOR STRONGLY-COUPLED PROBLEMS
}

\author{
R. Suliman ${ }^{1}$, O.F. Oxtoby ${ }^{1}$, A.G. Malan ${ }^{2}$, S. Kok ${ }^{3}$ \\ ${ }^{1}$ Advanced Computational Methods, Aeronautics Systems, Council for Scientific and Indus- \\ trial Research, Pretoria, 0002, South Africa \\ (rsuliman@csir.co.za)
}

${ }^{2}$ Department of Mechanical Engineering, University of Cape Town, Cape Town, 7700, South Africa

${ }^{3}$ Advanced Mathematical Modelling, Modelling and Digital Science, Council for Scientific and Industrial Research, Pretoria, 0002, South Africa

\begin{abstract}
This work details the development of a computational tool that can accurately model strongly-coupled fluid-structure interaction (FSI) problems, with a particular focus on thin-walled structures undergoing large, non-linear deformations. The first part of the work investigates improving the efficiency with which a stable and robust in-house code models thin structures undergoing dynamic fluid-induced bending deformations. Variations of the existing finite volume formulation as well as linear and higher-order finite element formulations are implemented. The governing equations for the solid domain are formulated in a total Lagrangian or undeformed conguration and large geometrically non-linear deformations are accounted for. As will be demonstrated, the finite volume approach exhibits similar disadvantages to the linear Q4 finite element formulation when undergoing bending. An enhanced finite volume approach is discussed and compared with finite element methods. The second part of this work is concerned with fluid-structure interaction (FSI) modelling. It considers the implementation and coupling of a higher-order finite element structural solver with an existing in-house fluid-flow solver. The coupling between the fluid and structural domains is rigorously assessed. The developed technology is validated through the simulation of representative two-dimensional strongly-coupled problems, on which rigorous mesh and temporal independence studies are also conducted. The results of three-dimensional FSI test-cases are also presented.
\end{abstract}

Keywords: fluid-structure interaction, finite volume, finite element 


\section{INTRODUCTION}

Computational mechanics is a growing discipline which uses computational methods to obtain approximate solutions to problems governed by the principles of mechanics. With the massive advances in computer technology over the past few decades, computational mechanics has become an important tool in analysing complex physical phenomena, and has had a significant influence on science and technology.

Since the 1960s, the finite element method has mainly been used for modelling the mechanics of solids [1]. The finite volume method [2] has traditionally been more dominant in the field of fluid mechanics but has also become increasingly popular for use in solid mechanics $[3,4,5,6,7,8]$. Both schemes can be considered as methods of weighted residuals where they differ in the choice of the weighting function. The finite element Galerkin method uses shape functions as the weighting functions, while the finite volume method results by choosing the weighting function as unity. Finite element methods are typically formulated in a total Lagrangian or undeformed configuration. In contrast, finite volume methods are traditionally based on an Eulerian or updated mesh configuration, which is not optimal for solid mechanics problems.

Fluid-structure interaction (FSI) constitutes a branch of computational mechanics in which there exists an intimate coupling between fluid and structural or solid domains; the behaviour of the system is influenced by the interaction of a moving fluid and a flexible solid structure. There are a wide variety of FSI problems encountered in many areas of aerospace, biomedical, mechanical and civil engineering. Examples of such problems include the prediction of wing flutter in aircraft [9] and arterial modelling in the human body [10]. Though much effort has been spent over recent years in developing FSI modelling technology, significant scope for improvement still exists in terms of industrial relevance and impact.

The purpose of this study is to furnish a computational tool that can accurately model strongly-coupled FSI problems, with a particular focus on thin-walled structures undergoing large, non-linear deformations.

\section{GOVERNING EQUATIONS}

The fluid-structure interaction system consists of a homogeneous isotropic elastic solid region and a viscous incompressible isothermal fluid domain. The set of equations describing each domain is now detailed.

\subsection{Solid equations}

The partial differential equations that describe a homogeneous isotropic elastic solid undergoing large geometrically non-linear deformation are given by Cauchy's first equation of motion (balance of linear momentum) [11], which in a total Lagrangian formulation or with respect to the undeformed configuration is

$$
\frac{\partial P_{i j}}{\partial X_{j}}+f_{i}=\rho_{o} a_{i},
$$


where $P_{i j}, f_{i}, \rho_{o}$ and $a_{i}$ are the first Piola-Kirchoff stress (a stress measure which relates forces in the deformed configuration with areas in the undeformed configuration), body force, density and acceleration, respectively and $X_{j}$ are the coordinates of a point on the solid body in the undeformed configuration.

In order to solve the elastic boundary value problem, Equation (1), a relationship between stress and displacement is required. This relation is obtained indirectly through the strain.

Assuming an isotropic hyperelastic St-Venant-Kirchoff material model, the GreenLagrange strain, $E_{i j}$, which is a strain measure in the undeformed configuration, is related to the stress by

$$
S_{i j}=\mathcal{C}_{i j k l} E_{i j}
$$

where $S_{i j}$ is the second Piola-Kirchoff stress and $\mathcal{C}_{i j k l}$ is the fourth order elasticity tensor.

For convenience, we can represent the stress and strain tensors in Equation (2) as vectors and the fourth order elasticity tensor as a matrix:

$$
\left\{\begin{array}{c}
S_{11} \\
S_{22} \\
S_{33} \\
S_{12} \\
S_{13} \\
S_{23}
\end{array}\right\}=b\left[\begin{array}{cccccc}
1 & \frac{\nu}{1-\nu} & \frac{\nu}{1-\nu} & 0 & 0 & 0 \\
\frac{\nu}{1-\nu} & 1 & \frac{\nu}{1-\nu} & 0 & 0 & 0 \\
\frac{\nu}{1-\nu} & \frac{\nu}{1-\nu} & 1 & 0 & 0 & 0 \\
0 & 0 & 0 & \frac{1-2 \nu}{1-\nu} & 0 & 0 \\
0 & 0 & 0 & 0 & \frac{1-2 \nu}{1-\nu} & 0 \\
0 & 0 & 0 & 0 & 0 & \frac{1-2 \nu}{1-\nu}
\end{array}\right]\left\{\begin{array}{c}
E_{11} \\
E_{22} \\
E_{33} \\
E_{12} \\
E_{13} \\
E_{23}
\end{array}\right\}
$$

where $b$ is a constant defined as

$$
b=\frac{E(1-\nu)}{(1+\nu)(1-2 \nu)}
$$

and $E$ is the Young's modulus and $\nu$ is the Poisson's ratio of the material.

Considering only two-dimensional cases, two possibilities exist to simplify the analysis. These are conditions of plane stress and plane strain. The plane stress condition exists when the body is very thin, i.e. in the limit where the third dimension approaches zero. Under such conditions Equation (3) simplifies to:

$$
\left\{\begin{array}{c}
S_{11} \\
S_{22} \\
S_{12}
\end{array}\right\}=\frac{E}{\left(1-\nu^{2}\right)}\left[\begin{array}{ccc}
1 & \nu & 0 \\
\nu & 1 & 0 \\
0 & 0 & 1-\nu
\end{array}\right]\left\{\begin{array}{c}
E_{11} \\
E_{22} \\
E_{12}
\end{array}\right\} .
$$

The plane strain condition exists when the body is very thick, i.e. in the limit where the third dimension approaches infinity. Equation (3) now becomes:

$$
\left\{\begin{array}{l}
S_{11} \\
S_{22} \\
S_{12}
\end{array}\right\}=\frac{E(1+\nu)}{(1-2 \nu)}\left[\begin{array}{ccc}
1-\nu & \nu & 0 \\
\nu & 1-\nu & 0 \\
0 & 0 & 1-2 \nu
\end{array}\right]\left\{\begin{array}{l}
E_{11} \\
E_{22} \\
E_{12}
\end{array}\right\} .
$$

The first Piola-Kirchoff stress, $P_{i j}$, in Equation (1) is then obtained by multiplying the second Piola-Kirchoff stress, $S_{k j}$, with the deformation gradient, $F_{i k}$ :

$$
P_{i j}=F_{i k} S_{k j}
$$


The deformation gradient relates quantities in the undeformed configuration to their counterparts in the deformed configuration:

$$
F_{i k}=\frac{\partial x_{i}}{\partial X_{k}}
$$

where $X_{k}$ and $x_{i}$ are the coordinates of the solid in the undeformed and deformed configurations, respectively.

Finally, to close the governing equations, the relationship between strain and the displacement field is given by

$$
E_{i j}=\frac{1}{2}\left(H_{i j}+H_{j i}+H_{k i} H_{k j}\right)
$$

where $H_{i j}$ is the displacement gradient defined as

$$
H_{i j}=\frac{d u_{i}}{d X_{j}}
$$

and $u_{i}$ is the total displacement of the solid, therefore $x_{i}=u_{i}+X_{i}$.

\subsection{Fluid equations}

The fluid flow is governed by the Navier-Stokes equations. In general, these equations are expressed in an Eulerian or spatial frame of reference, as opposed to a Lagrangian or material description for the solid, which entails a fixed spatial region with fluid flowing through it. For fluid-structure interaction problems the solid deforms and displaces the fluid domain and a mixture or combination of the two reference frames, referred to as an arbitraryLagrangian-Eulerian (ALE) reference frame, results for the motion of the FSI interface. This approach was first described by Hirt et al. [12] and later adopted by many others and is now widely used for FSI applications [13]. A dynamic mesh movement algorithm that deforms the fluid mesh is therefore required and is described later.

Assuming a viscous, incompressible and isothermal fluid, the equations governing the fluid flow are given by the continuity and Navier-Stokes equations:

$$
\begin{gathered}
\frac{\partial v_{i}}{\partial x_{i}}=0 \\
\rho \frac{\partial v_{i}}{\partial t}+\rho\left(v_{j}-v_{j}^{*}\right) \frac{\partial v_{i}}{\partial x_{j}}+\frac{\partial p}{\partial x_{i}}-\frac{\partial \sigma_{i j}}{\partial x_{j}}-\rho f_{i}=0
\end{gathered}
$$

where $\rho, v_{i}, p, \sigma_{i j}$ and $f_{i}$ are the fluid density, fluid velocity, pressure, stress and body forces respectively, $x_{i}$ are the fixed Eulerian coordinates and $v^{*}$ is the mesh velocity. The term

$\left(v_{j}-v_{j}^{*}\right)$ is an ALE convective velocity that results from a difference between the fluid velocity and the mesh velocity.

To close the fluid governing equations, a constitutive relation for the stress is required. Assuming a Newtonian fluid, the relationship between stress and rate of strain is given by

$$
\sigma_{i j}=\mu\left(\frac{\partial v_{i}}{\partial x_{j}}+\frac{\partial v_{j}}{\partial x_{i}}\right),
$$

where $\mu$ is the fluid viscosity. 


\section{GEOMETRICALLY NON-LINEAR STRUCTURES}

At the commencement of this study, a stable and robust in-house code, Elemental, was available [14]. With reference to FSI, the solver makes use of a compact edge-based vertexcentred finite volume method (FVM) to model both fluid and structure in a strongly-coupled partitioned manner. While this approach has been demonstrated to be effective for the fluid domain, thin structures require many elements through the thickness to ensure accuracy.

To improve the efficiency with which Elemental models thin structures undergoing bending deformations, variations of the existing finite volume method, as well as linear and higher-order standard Galerkin finite element methods (FEM) [15] are implemented. The various methods are compared with each other and the optimal approach chosen.

\subsection{Discretisation}

The standard Galerkin finite element method for discretisation of the solid governing equations is described in many handbooks $[15,16]$. Instead, we describe the finite volume method below.

Assuming the body forces, $f_{i}$, to be negligible and expressing the acceleration, $a_{i}$, as the rate of change of velocity, $v_{i}$, gives

$$
\rho_{o} \frac{\partial v_{i}}{\partial t}=\frac{\partial P_{i j}}{\partial X_{j}} .
$$

The equation is cast into integral or weak form by integrating over an arbitrary spatial subdomain $\mathcal{V}_{o}$ in the reference (undeformed) configuration:

$$
\int_{\mathcal{V}_{o}} \rho_{o} \frac{\partial v_{i}}{\partial t} d \mathcal{V}_{o}=\int_{\mathcal{V}_{o}} \frac{\partial P_{i j}}{\partial X_{j}} d \mathcal{V}_{o}
$$

The control volume, $\mathcal{V}_{o}$, is fixed in time, therefore differentiation and integration of the temporal term are interchangeable. In addition, $\rho_{o}$ is constant, so the left-hand-side of the equation simplifies to

$$
\rho_{o} \frac{d}{d t} \int_{\mathcal{V}_{o}} v_{i} d \mathcal{V}_{o}=\int_{\mathcal{V}_{o}} \frac{\partial P_{i j}}{\partial X_{j}} d \mathcal{V}_{o}
$$

Applying the divergence theorem of Gauss, the spatial derivative may be written in terms of fluxes as:

$$
\rho_{o} \frac{d}{d t} \int_{\mathcal{V}_{o}} v_{i} d \mathcal{V}_{o}=\oint_{\mathcal{A}_{m}} P_{i j} \cdot n_{j} d \mathcal{A}_{o}
$$

where $\mathcal{A}_{m}$ is the surface enclosing $\mathcal{V}_{o}$ and $n=\left(n_{1}, n_{2}\right)$ is the outward pointing unit-vector normal to $\mathcal{A}_{m}$.

In the standard edge-based vertex-centred finite volume method, the dependent variables are stored at nodes around which control volumes are constructed. In 2D, these control volumes are constructed by joining the midpoints of edges with element centroids and in such a way that only one node lies within each control volume. The set of surfaces forming the control volumes are referred to as a dual-mesh. This is shown schematically for a node $m$ in 
Figure 1 [17]. In the figure, $\mathcal{V}_{m}$ is the control volume associated with node $m$. Its bounding surface $\mathcal{A}_{m}$ is composed of a number of surfaces which are defined based on their associated edges. For example, $\mathcal{A}_{m n}$ is the surface segment intersecting the edge $\Upsilon_{m n}$ which connects nodes $m$ and $n$.

The surface integrals in Equation (17) are now calculated in an edge-wise manner, i.e. the surface integral is expressed as the sum over all the edges connecting the control volume:

$$
\rho_{o} \frac{d}{d t} \int_{\mathcal{V}_{o}} v_{i} d \mathcal{V}_{o}=\sum_{\Upsilon_{m n} \cap \mathcal{V}_{m}} P_{i j} \cdot C_{j: m n}+\sum_{\Upsilon_{m n}^{B} \cap \mathcal{V}_{m}} P_{i j} \cdot B_{j: m n}
$$

where $C_{j: m n}$ is the edge coefficients connecting arbitrary internal nodes $m$ and $n$ and $B_{j: m n}$ is the edge coefficient for an edge that lies along the volume boundary.

Discretising and integrating the temporal term on the left gives:

$$
\rho_{o} \frac{v_{i}^{\tau+\Delta \tau}-v_{i}^{\tau}}{\Delta \tau} \mathcal{V}_{o}=\sum_{\Upsilon_{m n} \cap \mathcal{V}_{m}} P_{i j} \cdot C_{j: m n}+\sum_{\Upsilon_{m n}^{B} \cap \mathcal{V}_{m}} P_{i j} \cdot B_{j: m n}
$$

where $\Delta \tau$ is the pseudo-timestep size.

To introduce the primary variable, displacement, into the equation, we simply note that velocity is the temporal rate of change of displacement, $u$,

$$
\frac{d u_{i}}{d t}=v_{i}
$$

The set of equations is solved via a single-step Jacobi iterative scheme [15] which is implemented such as to ensure a matrix-free and robust solution. Second-order-accurate temporal discretisation is achieved via dual-time-stepping [18], with both consistent and lumped mass matrices, and with a Jacobi pseudo-time iteration method employed for solver purposes. The matrix-free approach makes the scheme particularly well-suited for distributed memory parallel hardware architectures.

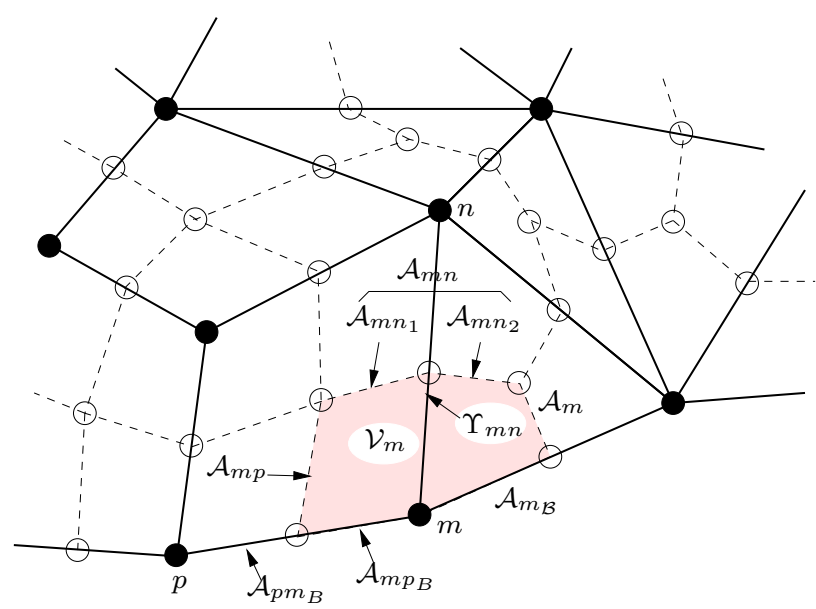

Figure 1. Schematic of the construction of a dual-mesh 


\subsection{Results}

To evaluate the finite volume and finite element methods in modelling bending, we consider a thick beam in pure bending (Figure 2). The beam was clamped at one end and subjected to a moment at the free end. The material properties used were a Young's modulus $E=210 \mathrm{GPa}$ and Poisson's ratio $\nu=0$. The length $l=6 \mathrm{~mm}$, height $h=1 \mathrm{~mm}$ and the plane stress assumption were used. The standard vertex-centred FVM was found to suffer from shear locking or sensitivity to element aspect ratio when subjected to bending (see Figure 3), which is a well-documented problem with the linear Q4 FEM [16]. To address this, an enhanced finite volume approach which uses and elemental-based displacement gradients [19] for the shear components of strain and node-based gradients for the normal components, was implemented. This hybrid FVM approach is shown to be insensitive to element aspect ratio, as can be seen in Figure 3.

A comparison between the FVM and FEM on a geometrically non-linear structure in bending is shown in Figure 4. Although the hybrid FVM exhibits distinct advantages over the Q4 FEM, the higher-order Q8 FEM produced the most desirable results.

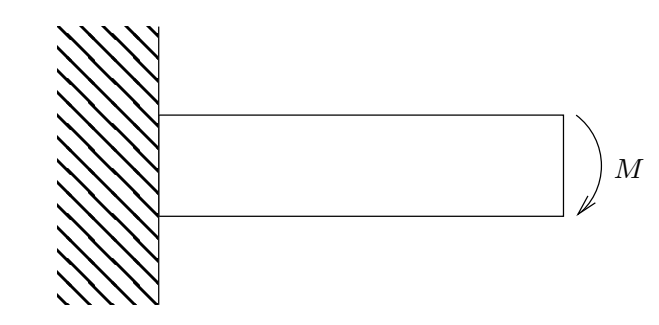

Figure 2. Cantilever beam in pure bending

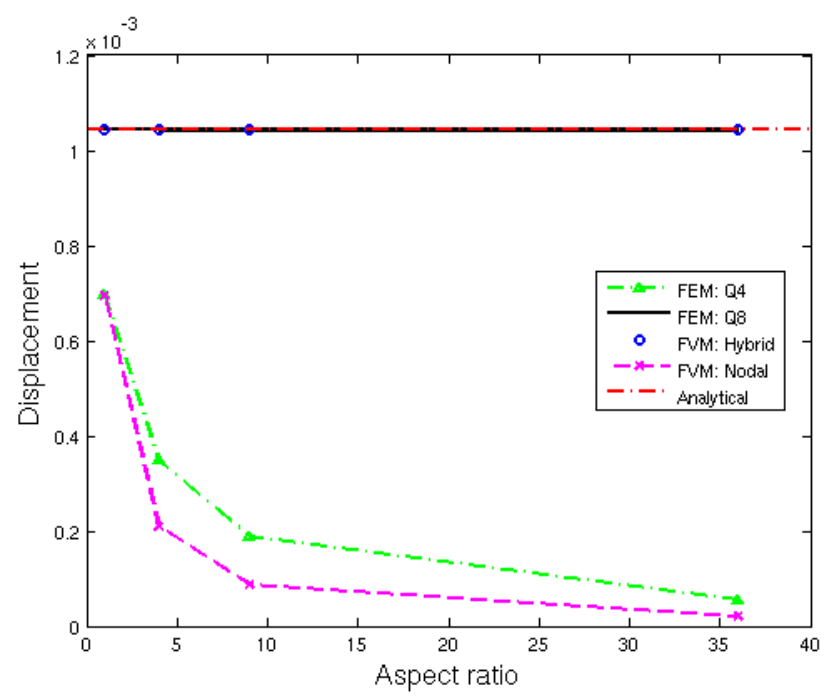

Figure 3. Graph depicting shear-locking mechanism present in the Q4 FEM and standard vertex-centred FVM when subjected to bending 


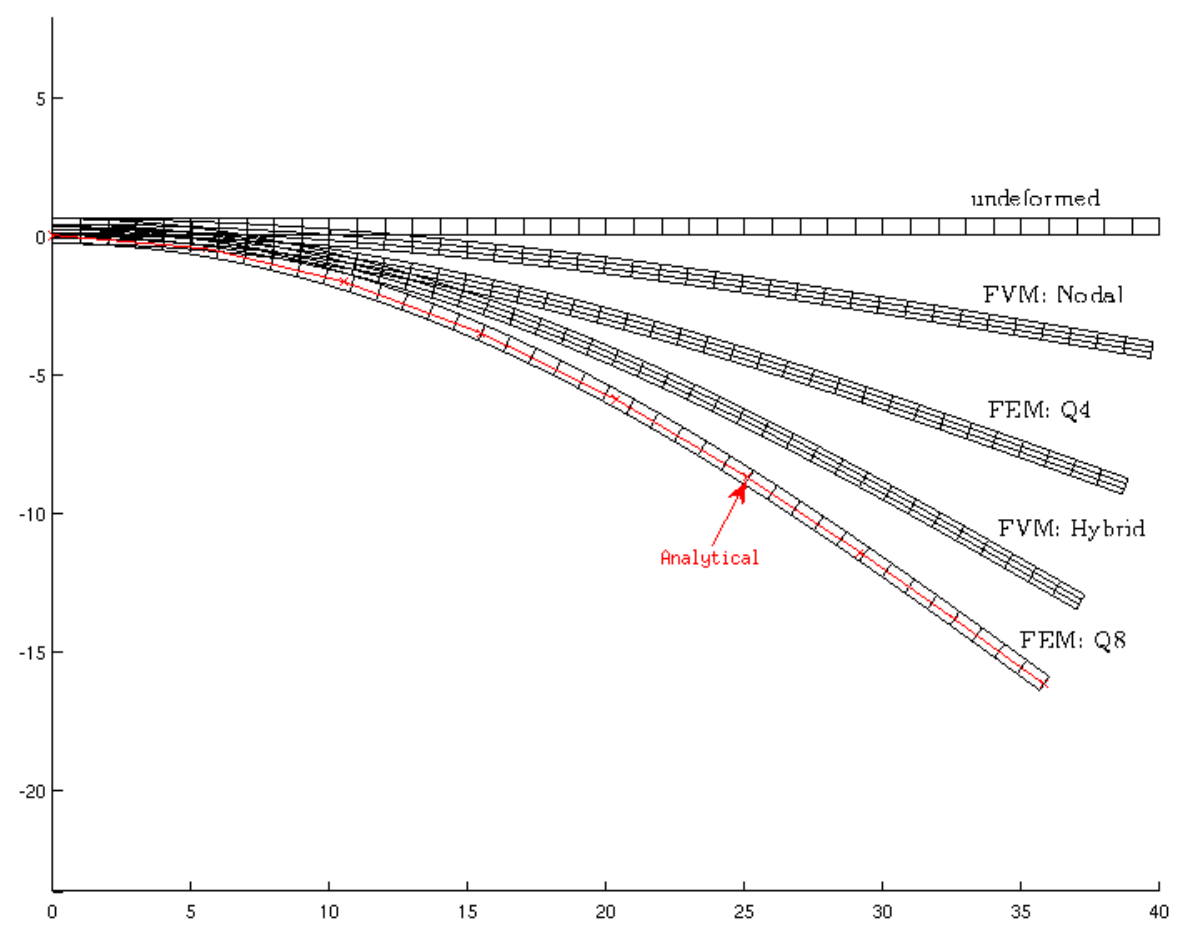

Figure 4. Comparison of FVM and FEM on a geometrically non-linear beam in bending

\section{FLUID-STRUCTURE INTERACTION}

The second part of this study looks at the implementation and coupling of the Q8 FEM solid mechanics solution procedure with the existing fluid-flow solver in Elemental. A hybrid finite volume-finite element FSI scheme is developed.

\subsection{Fluid-solid interface treatment}

The fluid-structure interaction system can be described in general or broad terms as follows: the fluid flow provides a traction or load onto the structure. This results in a dynamic response or deformation of the structure which in turn affects the geometry of the fluid domain and the fluid flow. In order to solve this intimately coupled system, the following coupling conditions for the traction, displacement and velocity need to be satisfied at the fluid-solid interface:

$$
\begin{aligned}
t_{i}^{s} & =p n_{i}-\sigma_{i j}^{f} n_{j} \\
u_{i}^{f} & =u_{i}^{s} \\
v_{i}^{f} & =v_{i}^{*}=v_{i}^{s}
\end{aligned}
$$

where $n_{i}$ is the related unit vector pointing outward from the fluid domain, the superscripts $f$ and $s$ refer to the fluid and solid respectively. Equation (21) represents momentum conservation or force equilibrium at the interface, while Equations (22) and (23) enforce the 
kinematic or geometric continuity and no-slip conditions respectively. Through the use of dual-timestepping, the transfer of information between the fluid and solid is done at solver sub-iteration level and strong-coupling is effected through the procedure described next.

\subsection{Mesh movement and solution procedure}

The movement of the fluid mesh is handled via an interpolation procedure. In this approach the closest internal and external boundary nodes for every internal fluid node are identified and the distances between the boundary nodes and the internal node computed at the beginning of the analysis. The movement of internal fluid nodes is then determined by means of an interpolation function that uses the displacements of the closest boundary nodes. The reader is referred to [20] for further details.

The solution procedure for the coupled FSI problem involves solving the discretised fluid and solid governing equations and updating the mesh in an iterative manner that effects strong coupling, as follows:

1. The fluid governing equations, Equations (11) to (13), are solved for an iteration. This solution provides tractions that are applied to the solid domain.

2. The solid discrete equations, Equations (19) and (20), are then solved for an iteration, which provides velocities and displacements at the boundary of the FSI interface. The boundary velocities are applied to the fluid mesh, which results in an ALE convective velocity in the fluid domain.

3. The mesh is moved if the displacement of a solid mesh boundary node exceeds $30 \%$ of the element size or the residuals of the fluid and solid discrete equations have decreased by at least four orders of magnitude.

4. The steps above are repeated until convergence, i.e. the residuals of fluid and solid discrete equations are calculated and if greater than the convergence tolerance, Steps 1 to 3 are repeated. If the residuals have decreased by at least five orders of magnitude, the real-timestep is terminated and the next timestep is entered by proceeding to Step 1.

\subsection{Results}

The developed technology is evaluated via application to benchmark two-dimensional strongly-coupled large-displacement FSI test problems from literature. A popular test-case is that of a thin elastic beam attached to a rigid square block with fluid flowing over it [21, 22]. The geometry and boundary conditions are shown in Figure 5. The properties of the incompressible fluid are: density $\rho_{f}=1.18 \times 10^{-3} \mathrm{~g} \mathrm{~cm}^{-3}$ and viscosity $\mu_{f}=1.82 \times 10^{-4}$ $\mathrm{g} \mathrm{cm}^{-1} \mathrm{~s}^{-1}$, while that of the beam are: density $\rho_{s}=2.0 \mathrm{~g} \mathrm{~cm}^{-3}$, Young's modulus $E=$ $2.0 \times 10^{6} \mathrm{~g} \mathrm{~cm}^{-1} \mathrm{~s}^{-2}$ and Poisson's ratio $\nu=0.35$. The plane stress assumption was used. A

uniform constant fluid velocity, $v_{\text {in }}=31.5 \mathrm{~cm} \mathrm{~s}^{-1}$, or Reynolds number, $R e=\frac{\rho_{f} L v_{i n}}{\mu_{f}}=204$, was applied at the inlet while at the exit the pressure was set to zero. The inlet velocity results in unsymmetric vortices on either side of the beam which eventually build up causing large 


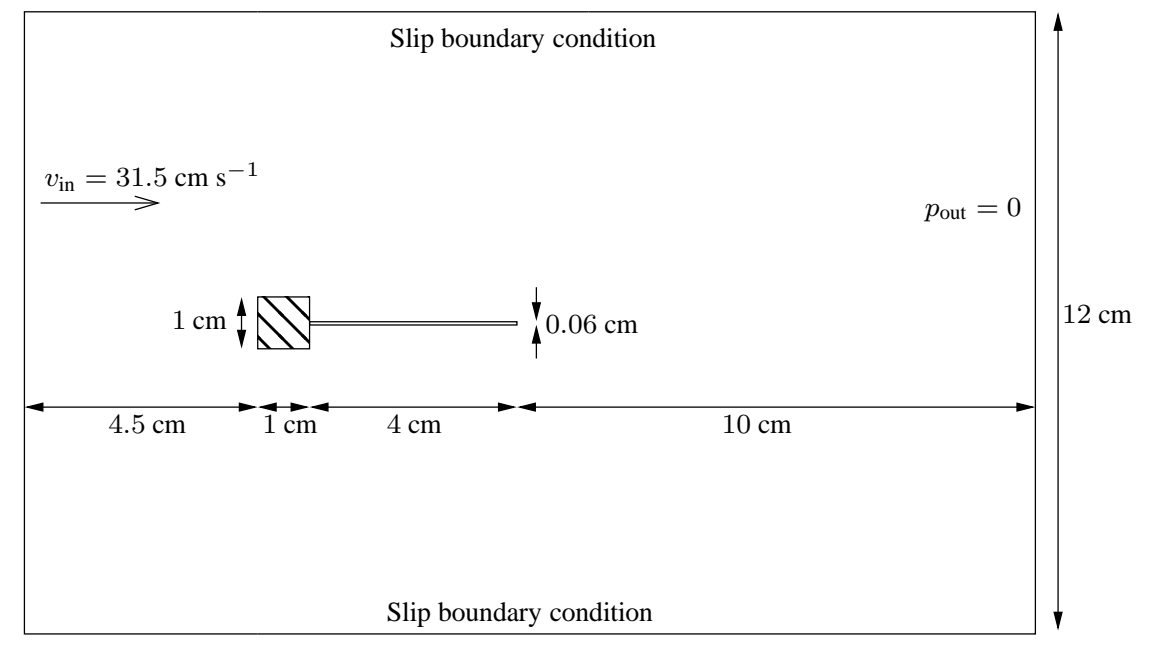

Figure 5. Geometry and boundary conditions for the block-tail FSI test-case
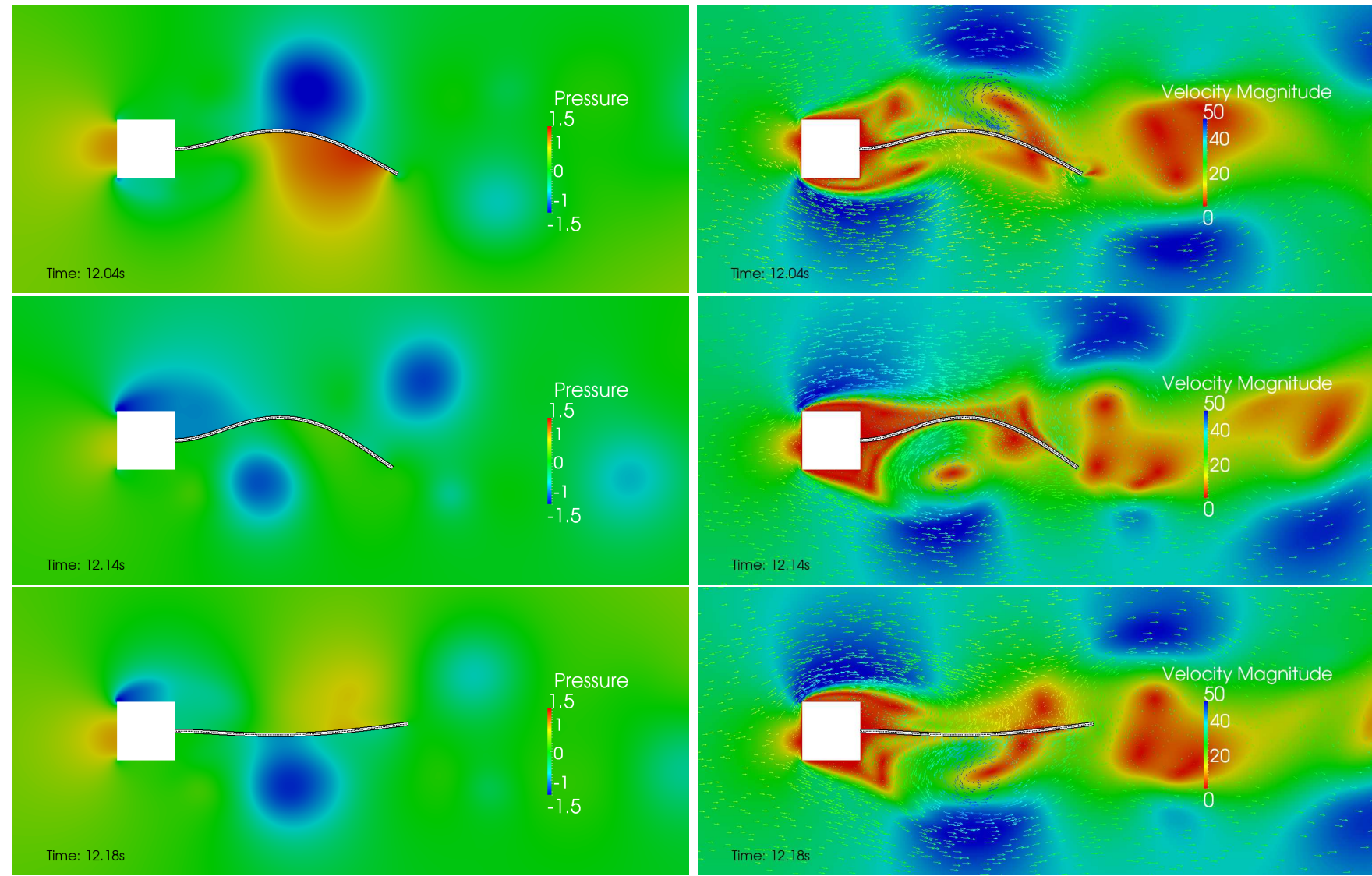

Figure 6. Pressure (left) and velocity contours (right) for the block-tail FSI test-case with the beam oscillating in its second mode of vibration 
oscillations of the beam in its second mode of vibration. Plots of the pressure and velocity contours are shown in Figure 6.

The time history of the tip displacement of the beam is plotted in Figure 7(a), together with the results of Hubner et al. [22]. There is a good correlation between the two sets of results, with the amplitude and frequency differing by less than $3 \%$ and $4 \%$ respectively as shown in Table 1. A rigorous mesh and temporal independence study was conducted. To evaluate mesh independence of the fluid two finer meshes of 25000 and 50000 fluid elements were generated. As can be seen in Figure 7(b) and Table 1, a small change in solution is observed from the 6000 to the 25000 element mesh, but a further doubling of the number of elements results in a negligible difference. Next, to evaluate mesh independence of the solid a finer solid mesh of $80 \times 2$ Q8 elements was used and it was found that the results are identical to the $40 \times 1$ solid mesh case (see Figure 7(c) and Table 1). Finally, temporal independence was evaluated by using four different timestep sizes varying from $0.005 \mathrm{~s}$ to $0.0005 \mathrm{~s}$. The time history in Figure 7(d) as well as the result in Table 1 show good convergence and independence in the results.

This partitioned hybrid FSI scheme was extended to solve two strongly-coupled 3D problems. These include a pressure pulse in a flexible tube and a flapping elastic membrane. Snapshots of these results are shown in Figure 8.

\section{CONCLUSIONS}

A vertex-centred and novel hybrid finite volume method to model geometrically nonlinear solid mechanics was investigated and compared with the traditional finite element method in this work. The higher-order Q8 finite element method produced the most desirable results and was coupled to an existing fluid-flow solver to develop a hybrid finite volume-finite element fluid structure interaction scheme. The scheme was evaluated on strongly-coupled benchmark problems, demonstrating full coupling between fluid and structural domains, while furnishing accurate results.

\begin{tabular}{|ccc|cc|}
\hline Fluid mesh & Solid mesh & Timestep $(\mathrm{s})$ & Amplitude $(\mathrm{cm})$ & Frequency $(\mathrm{Hz})$ \\
\hline 6000 & $40 \times 1$ & 0.001 & 0.78 & 2.98 \\
6000 & $80 \times 2$ & 0.001 & 0.78 & 2.98 \\
25000 & $120 \times 1$ & 0.001 & 0.76 & 2.97 \\
50000 & $160 \times 1$ & 0.001 & 0.76 & 2.97 \\
6000 & $40 \times 1$ & 0.0005 & 0.78 & 2.97 \\
\hline \multicolumn{3}{|c|}{ Hubner et al. [22] } & 0.8 & 3.1 \\
\hline Xia et al. [19] & 0.81 & 3.3 \\
\hline
\end{tabular}

Table 1. Comparison of amplitude and frequency for the block-tail test-case in second mode of vibration with various meshes and timestep sizes 


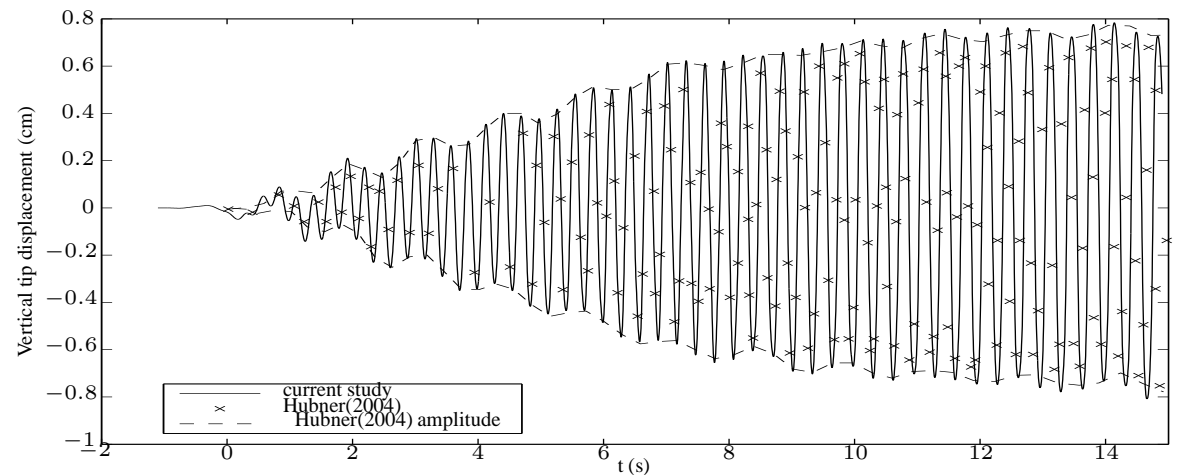

(a)

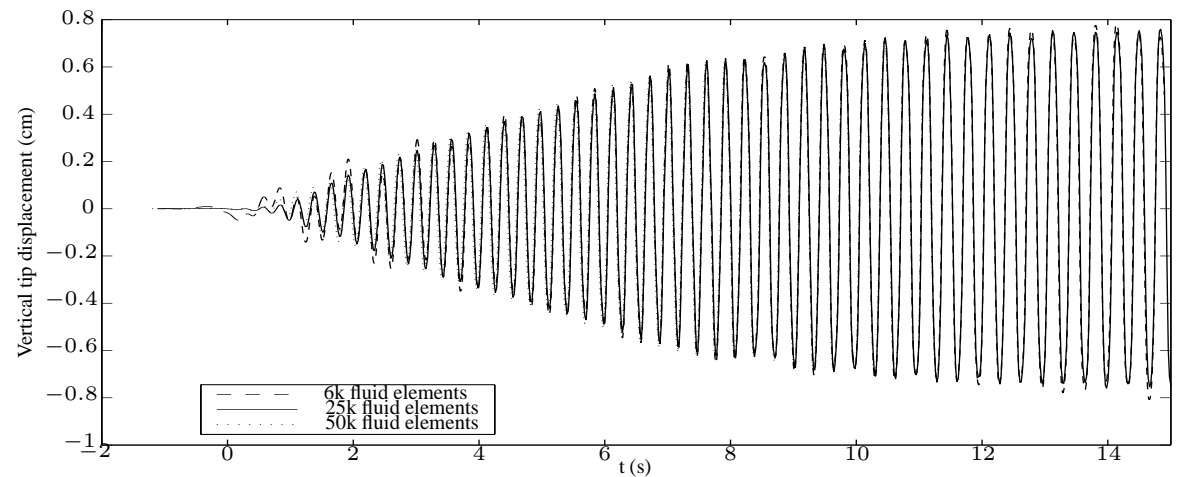

(b)

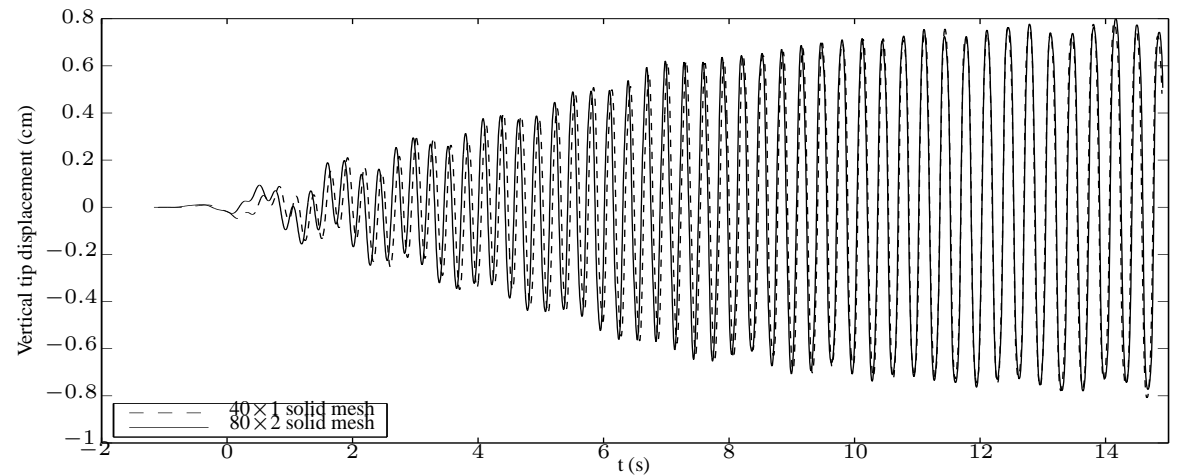

(c)

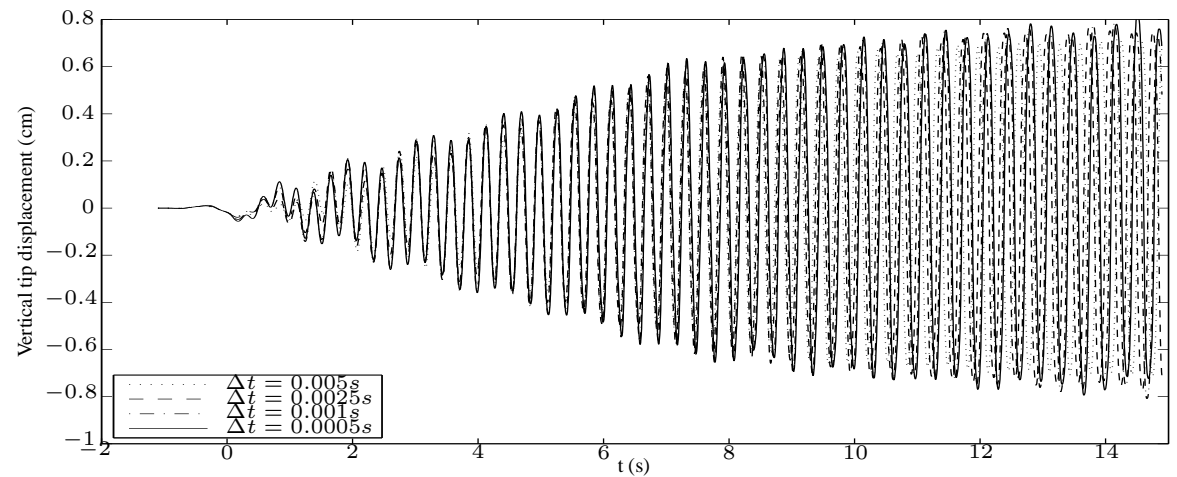

(d)

Figure 7. Tip displacement for the block-tail test-case in second mode of vibration with various meshes and timestep sizes 

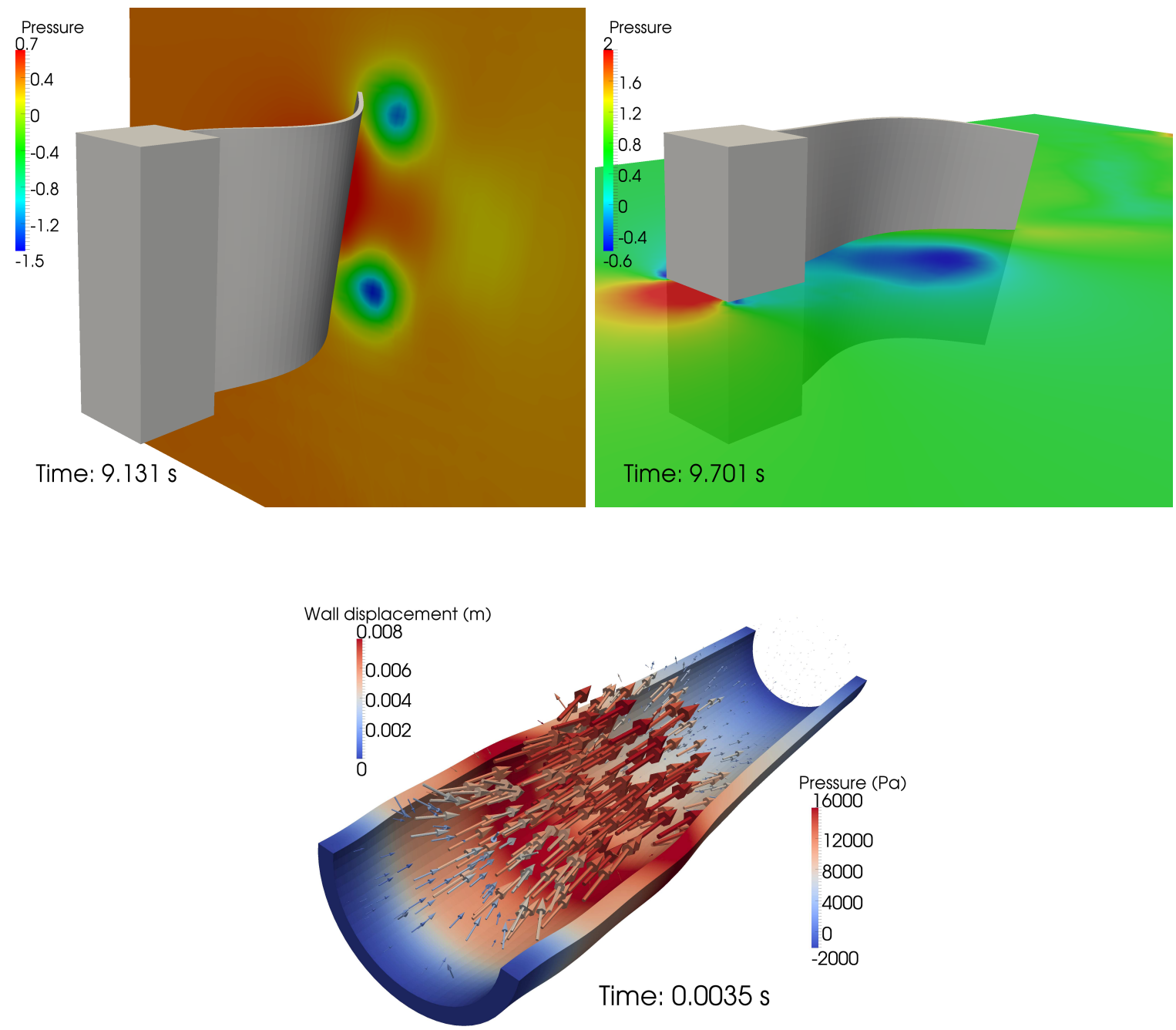

Figure 8. Pressure contours for flapping membrane (top) and pipe displacement and velocity vectors for pressure pulse propagating in a flexible tube (bottom) 


\section{REFERENCES}

[1] O. C. Zienkiewicz and R. L. Taylor. The Finite Element Method: Volume 2 - Solid Mechanics. Butterworth-Heinemann, Oxford, fifth edition, 2000.

[2] S. V. Patankar. Numerical Heat Transfer and Fluid Flow. McGraw-Hill, New York, 1980.

[3] E. Oñate, M. Cervera, and O. C. Zienkiewicz. A finite-volume format for structural mechanics. International Journal for Numerical Methods in Engineering, 37:181-201, 1994.

[4] M.A. Wheel. A finite-volume approach to the stress analysis of pressurized axisymmetric structures. International Journal of Pressure Vessels and Piping, 68:311-317, 1996.

[5] J. Fainberg and H.-J. Leister. Finite volume multigrid solver for thermo-elastic stress analysis in anisotropic materials. Computer Methods in Applied Mechanics and Engineering, 137:167-174, 1996.

[6] M.A. Wheel. A finite volume method for analysing the bending deformation of thick and thin plates. Computer Methods in Applied Mechanics and Engineering, 147:199-208, 1997.

[7] A.K. Slone, C. Bailey, and M. Cross. Dynamic solid mechanics using finite volume methods. Applied Mathematical Modelling, 27:69-87, 2003.

[8] N. Fallah. A cell vertex and cell centred finite volume method for plate bending analysis. Computer Methods in Applied Mechanics and Engineering, 193:3457-3470, 2004.

[9] A.K. Slone, K. Pericleous, C. Bailey, M. Cross, and C. Bennett. A finite volume unstructured mesh approach to dynamic fluid-structure interaction: an assessment of the challenge of predicting the onset of flutter. Applied Mathematical Modelling, 28:211239, 2004.

[10] C.A. Taylor, T.J.R. Hughes, and C.K. Zarins. Finite element modelling of blood flow in arteries. Computer Methods in Applied Mechanics and Engineering, 158:155-196, 1998.

[11] G.A. Holzapfel. Nonlinear Solid Mechanics: A continuum Approach for Engineering. John Wiley \& Sons Ltd., West Sussex, England, 2001.

[12] C.W. Hirt, A.A. Amsden, and J.L. Cook. An arbitrary Lagrangian-Eulerian computing method for all flow speeds. Journal of Computational Physics, 14:227-253, 1974.

[13] K.J. Bathe and G.A. Ledezma. Benchmark problems for incompressible fluid flows with structural interactions. Computers and Structures, 85:628-644, 2007. 
[14] A.G. Malan and R.W. Lewis. An artificial compressibility CBS method for modelling heat transfer and fluid flow in heterogeneous porous materials. International Journal for Numerical Methods in Engineering, 87:412-423, 2011.

[15] O. C. Zienkiewicz and R. L. Taylor. The Finite Element Method: Volume 1 - The Basis. Butterworth-Heinemann, Oxford, fifth edition, 2000.

[16] R.D. Cook, D.S. Malkus, M.E. Plesha, and R.J. Witt. Concepts and Applications of Finite Element Analysis. John Wiley \& Sons Ltd., United States, fourth edition, 2002.

[17] A. G. Malan. Investigation into the Continuum Thermodynamic Modeling of Investment Casting Shell-Mould Drying. PhD thesis, University of Wales Swansea, 2002.

[18] V. Venkatakrishnan and D. J. Mavriplis. Implicit method for the computation of unsteady flows on unstructured grids. Journal of Computational Physics, 127:380-397, 1996.

[19] G. Xia and C.-L. Lin. An unstructured finite volume approach for structural dynamics in response to fluid motions. Computers and Structures, 86:684-701, 2008.

[20] O.F. Oxtoby, A.G. Malan, and P. Nithiarasu. A novel matrix free, edge based finite volume method for partitioned solution of fluid-structure interaction problems. International Journal for Numerical Methods in Engineering, submitted for review.

[21] W. Wall. Fluid-struktur-interaktion mit stabilisierten finiten elementen. $\mathrm{PhD}$ thesis, Universitat Stuttgart, 1999.

[22] B. Hubner, E. Walhorn, and D. Dinkler. A monolithic approach to fluid-structure interaction using space-time finite elements. Computer Methods in Applied Mechanics and Engineering, 193:2087-2104, 2004. 